Material, radius of curvature $(r)$ and focal length $(f)$ of biconcave lens for 8-keV X-ray

\begin{tabular}{lrr} 
Material & $r(\mathrm{~mm})$ & $f(\mathrm{~m})$ \\
Gold & 10 & 100 \\
Gold & 5 & 50 \\
Platinum & 10 & 90 \\
Tungsten & 10 & 100 \\
\hline
\end{tabular}

shows the materials, the radii of curvature and the focal lengths for biconcave lenses. Because the lens is concave, we can reduce the thickness to a few micrometres by using a Fresnel lens, which can be made with an ultra-fine lathe. The intensity reduction due to absorption, therefore, may be a factor of only $1 / 10$. Parasitic, fluorescent X-rays from the lens diverge isotropically; we can eliminate them by conventional slits.

The advantages of lenses over mirrors are as follows. (1) The instability of the principal axis of the lens relative to the beam direction does not give rise to serious change in the position of the focal image of the light source. (2) The parasitic scattering due to the surface roughness of a lens is reduced to a factor of $\delta\left(10^{-6}\right)$ compared with that for a mirror with the same roughness. (3) The lens is much smaller than a mirror; to get a beam $1 \mathrm{~mm}$ in diameter, a totally reflecting mirror is typically $10 \mathrm{~cm}$ in length, whereas a lens needs to be only a few millimetres in diameter. No bending mechanism is required for lenses. (4) As the lens is made of metal, cooling efficiency is high. This is an important feature of optical devices for strong synchrotron radiation. (5) The beam path is straight in the lens system; a totally reflecting mirror causes deviation of the focused position from the straight beam path by a few tens of metres for a $1-\mathrm{km}$ beam line.

Another possible application of X-ray lenses may be microscopy. For soft $X$ rays of $10 \mathrm{~nm}$ in wavelength, $\delta$ is about $10^{-2}$ for graphite; we can adopt concave lenses for X-ray microscopy instead of zone plates.

Department of Polymer Chemistry,

Faculty of Engineering,

Kyoto University,

Kyoto 606, Japan

Department of Physics,

H. MIYAII*

Faculty of Science,

Kyoto University,

Kyoto 606, Japan

Department of Materials Chemistry,

Faculty of Science and Technology,

Ryukoku University,

Seta, Otsu 520-21, Japan

1. Kirkpatrick, P. \& Pattee H. H. Jr in Encyclopedia of Physics 30 (ed. Flugge, S.) 322-324 (Springer, Berlin 1957).

2. Compton, A. H. in X-rays and Electrons 205-220 (van Nostrand, New York, 1926).

* To whom correspondence should be addressed.

\title{
Bayesian reasoning in science
}

SIR - C. Howson and P. Urbach in their Commentary on bayesian reasoning in science (Nature 350, 371-374; 1991) state: "It might be imagined that a 95 per cent confidence interval corresponds to a 0.95 probability that the unknown paramater lies in the confidence range. But in the classical approach $\mu$ is not a random variable, and so has no probability. Nevertheless, statisticians regularly say that one can be ' 95 per cent confident' that the parameter lies in the confidence interval."

It seems that this problem can be solved by saying: the 95 per cent confidence interval covers $\mu$ with a probability of 95 per cent.

\section{Institut für Strahlenbiologie, Universität Münster, \\ Hittorfstraße 17, \\ 4400 Münster, Germany}

SIR - Howson and Urbach's eloquent contribution $^{1}$ to the literature intended to persuade scientists to adopt bayesian methods of statistical inference does not fall on deaf ears, but rather on ears eager to hear any fresh argument that could dispel the doubts repeatedly expressed in the 140 years since the time of inference, the only example he gave of the application of his famous (nonbayesian) procedure involved drawing balls out of an urn. Nor should the name of Thomas Bayes be too closely associated with modern bayesian arguments: that cautious man not only (like Bernoulli) made no attempt to publish his work during his lifetime, but his advocacy of the use of a prior distribution was tentative.

Unsurprisingly, the application of the probability axioms to scientific hypotheses leads to serious difficulties concerning the prior distributions to be adopted in the absence of information, as may be demonstrated using an example of Fisher's. A homozygous black mouse $(B B)$ when mated to a heterozygous black $(B b)$ will produce only black mice, but half will be expected to be homozygotes and half heterozygotes. An offspring of such a mating, therefore, may be stated, before progeny-testing, to be homozygous with probability one-half.

Now a bayesian statistician, confronted with a black mouse about whose ancestry he knows nothing (and whose ignorance of mendelian genetics is, in any case, complete) is constrained by his axioms to assert that, because he is unable to see any reason why the black mouse should be of one genotype rather than the other, the mouse is homozygous with probability one-half. Thus the bayesian's assertion, based on ignorance, is exactly the same as the scientist's, based on partial knowledge.

Fisher ${ }^{2}$ remarked that "It is evidently easier for the practitioner of natural science to recognize the difference between knowing and not knowing than this seems to be for the more abstract mathematician", adding that "no experimenter would feel he had a warrant for arguing as if he knew that of which in fact he was ignorant". As Boole ${ }^{3}$ had remarked in 1854, the appearance of arbitrary constants reflecting the prior assumptions of bayesian theory "seems to imply, that definite solution is impossible, and to mark the point where inquiry ought to stop".

Though I hold no brief for the 'repeated-sampling' theories of statistical inference about which Howson and Urbach are critical, in one example they are unfairly so. They assert that statisticians "never say why one can be ' 95 per cent confident' that the parameter lies in the confidence interval" in the case of the unknown mean $\mu$ of a normal distribution with known (not estimated) standard deviation of the mean $s$ and observed mean $m$, for which the confidence interval is $m-1.96 \mathrm{~s} \leqslant \mu \leqslant m+$ $1.96 \mathrm{~s}$

The reason was given by Fisher in the early 1930 s. He pointed out (1) that if 